\title{
Creep in Wood Under Variable Climate Conditions: Numerical Modeling and Experimental Validation
}

\author{
F. DUBOIS, H. RANDRIAMBOLOLONA and C. PETIT \\ Laboratory of Mechanics and Modelling of Materials and Structures in Civil Engineering, \\ University of Limoges, IUT Génie Civil, 19300 Egletons, France; \\ (E-mail:dubois@egletons.unilim.fr)
}

(Received 27 November 2002; accepted in revised form 8 July 2005)

\begin{abstract}
This paper deals with the modeling of linear viscoelastic behavior and strain accumulation (accelerated creep) during moisture content changes in timber. A generalized Kelvin-Voigt model is used and associated in series with a shrinkage-swelling element depending on the mechanical and moisture content states of materials. The hygrothermal aging due to climatic variations implies an evolution of rheological parameters depending upon moisture content and temperature. Two distinct viscoelastic laws, one for drying and the other for moistening, are coupled according to the thermodynamic principles when wood is subjected to nonmonotonous moisture variations. An incremental formulation of behavior is established in the finite element program CAST3M (Software developed by C.E.A. (Commissariat á l'Energi Atomique) and an experimental validation from tension creeprecovery tests is presented.
\end{abstract}

Key words: aging, creep, generalized Kelvin-Voigt model, mechanosorption, moisture content, temperature, thermodynamics, viscoelasticity

\section{Introduction}

Wood is a porous, hygroscopic, anisotropic and nonhomogenous biopolymer of cellular structure. It is classified as a viscoelastic material with mechanical properties depending on temperature and moisture content (Cariou, 1987; Genevaux and Guitard, 1988). The latter has indeed a prevalent effect compared to temperature. Creep's effects are an important factor for the design and the durability of timber structures. The evolution of creep results from the interactions between the mechanical stress and the internal moisture whose variations cause its acceleration. Much work has been done on the subject since the early 1960s (Armstrong and Kingston, 1960). A review of work was given by Grossman (1976), Morlier (1994), and Hunt (1999). The phenomenon called "Mechanosorption" has a chemical origin partially linked to the behavior of molecular bonds but the real mechanism is not yet well established. Phenomenological models are developed but they are often validated for a specific type of load (Ranta-Maunus, 1975; Bazant, 1985; Mukuday and Yata, 1986; Gril, 1988; Hunt, 1988; Toratti, 1992; Martensson, 1992; Salin, 1992; Hanhijarvi, 2000) and are not easily generalized for other conditions or problems. 
Our objective is to model the viscoelastic behavior of wood according to the thermodynamic principles. This viscoelastic approach allows a satisfying decoupling of the different phenomena during creep under variable climate conditions.

In the first section, a new hygromechanical numerical modeling of wood viscoelasticity is presented, integrating the hereditary effects (strain-stress history) and the environmental effects (moisture content and temperature). Based on the thermodynamic principles, the environmental effects are taken into account in the generalized Kelvin-Voigt model. The second section deals with an incremental formulation, implemented in a finite element program, which is obtained by integrating differential equations of models with a finite time difference method.

In the third section, an experimental validation is proposed from creep-recovery tests carried out by Pittet (1996) on thin specimens subjected to a longitudinal tension loading and moisture variations.

\section{Generalized Kelvin-Voigt Model}

In order to introduce environmental effects (hygrothermal aging) in viscoelastic behavior, we propose a rheological approach adapted to wood material.

\subsection{Constitutive Law: Rheological Approach}

In the classical treatment of the aging linear viscoelastic behavior (Bazant et al., 1999), the stress-strain relationship is given by the classical Stiljes-Volterra integral:

$$
\underline{\underline{\varepsilon}}(t)=\int_{0^{-}}^{t} \mathbf{J}(t, \tau) \frac{\partial \stackrel{\sigma}{\partial}}{\partial \tau} d \tau \quad \text { or } \quad \underline{\underline{\sigma}}(t)=\int_{0^{-}}^{t} \mathbf{R}(t, \tau) \frac{\partial \underline{\underline{\varepsilon}}}{\partial \tau} d \tau
$$

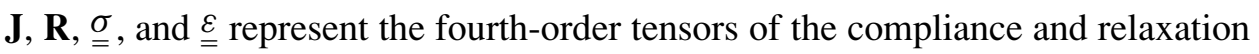
functions and the second-order stress and strain tensors, respectively. In a nonaging case, the compliance and relaxation functions are reduced to $\boldsymbol{J}(t, \tau)=\boldsymbol{J}(t-\tau)$ and $\boldsymbol{R}(t, \tau)=\boldsymbol{R}(t-\tau)$. Some authors admit a power law (or parabolic law) of the nonaging creep evolution (Cariou, 1987; Huet, 1988; Le Govic et al., 1988). This interpolation presents the advantage of obtaining a compliance tensor with few parameters to be identified. However, this technique is accurate enough for limited times and prohibits physical and thermodynamic interpretations of behavior. In this context, we prefer a rheological model consisting of springs and dashpots developed from a more rigorous thermodynamic approach. The generalized Maxwell model requires an identification based on relaxation tests. For wood material, creep tests lead us to opt for the generalized Kelvin-Voigt model. In order to simplify the presentation, we propose in Figure 1 a uniaxial model, where $\sigma$ and $\varepsilon$ are uniaxial stress and strain, respectively. 


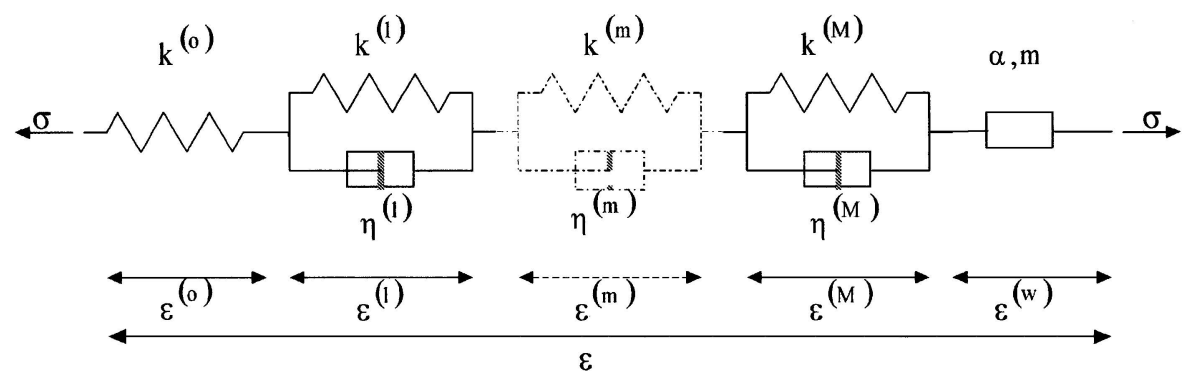

Figure 1. Generalized Kelvin-Voigt model in series with a moisture induced shrinkageswelling element.

In order to take into account all the phenomena during the creep of wood in variable climate conditions, our model is composed of an isolated spring representing the instantaneous response $\varepsilon^{(0)}$. Kelvin-Voigt cells define the primary creep $\varepsilon^{(m)}$. According to models with combined activation, (Hunt, 1999; Bazant, 1985; Gril, 1988), all springs and dashpots properties have a moisture content dependence. On the other hand, the last element represents moisture-induced shrinkage-swelling $\varepsilon^{(w)}$ depending on mechanical and moisture states of material. This last effect is assumed to be independent like models with independent activation, (Leicester, 1971; Ranta-Maunus, 1975; Hunt, 1988).

The total strain $\varepsilon(t)$ is then written in the following form:

$$
\varepsilon(t)=\varepsilon^{(0)}(t)+\sum_{m=1}^{M} \varepsilon^{(m)}(t)+\varepsilon^{(w)}(t)
$$

The viscoelastic strain $\varepsilon^{(\mathrm{ve})}=\varepsilon^{(0)}+\sum_{m=1}^{M} \varepsilon^{(m)}$ is the strain of the generalized Kelvin-Voigt model. In the uniaxial case of nonaging linear viscoelastic behavior, ${ }^{1}$ Volterra's integral (1) is written as follows:

$$
\varepsilon^{(\mathrm{ve})}(t)=\int_{0^{-}}^{t} J(t-\tau) \frac{\partial \sigma}{\partial \tau} d \tau
$$

with $J(t-\tau)=\frac{1}{k^{(0)}}+\sum_{m=1}^{M} \frac{1}{k^{(m)}}\left(1-e^{-\lambda^{(m)}(t-\tau)}\right), \quad \lambda^{(m)}=\frac{k^{(m)}}{\eta^{(m)}}$

The spring modulus noted $k^{(p)}, p \in(0,1, \ldots, m, \ldots, M)$ and dashpot viscosities noted $\eta^{(q)}, q \in(1, \ldots, m, \ldots, M)$ must be constant and positive.

The aging phenomenon appears as a variation of mechanical characteristics (stiffness, viscosity, etc., ...) over time at the macro-level of material description. For wood, the aging origin is due to the climate variation over time. Thus, the characteristics of each rheological element depend on moisture content at constant temperature. The modulus of aging spring $k^{(p)}(w)$ is defined from the function 
$b^{(p)}(w)$ and the modulus reference $k_{\text {ref }}^{(p)}$ corresponds to moisture content reference $w_{\text {ref }}$ such as:

$$
\begin{aligned}
& k^{(p)}(t)=b^{(p)}(t) k_{\text {ref }}^{(q)} \quad \text { with } \\
& b^{(p)}(w(t))=b^{(p)}(t), \quad b^{(p)}\left(w_{\text {ref }}\right)=1, \quad k_{\text {ref }}^{(p)}>0
\end{aligned}
$$

The wood's overall stiffness increases (hardening) during water desorption and decreases (softening) during absorption. The function $\dot{b}^{(p)}(t)$ is thus positive with drying and negative with moistening. In the same way, the aging dashpot's viscosity $\eta^{(q)}(w)$ depends on moisture content $w$ through a function $a^{(q)}(w)$ and a viscosity reference $\eta_{\text {ref }}^{(q)}$ associated with the moisture content reference $w_{\text {ref }}$ such as:

$$
\begin{aligned}
\eta^{(q)}(t) & =a^{(q)}(t) \eta_{\text {ref }}^{(q)} \quad \text { with } \\
a^{(q)}(w(t)) & =a^{(q)}(t), \quad a^{(q)}\left(w_{\text {ref }}\right)=1, \quad \eta_{\text {ref }}^{(p)}>0
\end{aligned}
$$

\subsection{THERMODYNAMIC RESTRICTIONS}

The compliance function $J(t, \tau)$ is thermodynamically admissible if thermodynamic laws are satisfied. At macroscopic level, it is translated by the conditions:

$$
J(t, \tau) \geq 0, \quad \frac{\partial J(t, \tau)}{\partial t} \geq 0, \quad \frac{\partial J(t, \tau)}{\partial t} \leq 0 \quad \text { and } \quad \frac{\partial^{2} J(t, \tau)}{\partial t \partial \tau} \leq 0
$$

Thermodynamic laws are checked against the compliance function $J(t, \tau)$ if they are also satisfied for the rheological model. Nevertheless, if the thermodynamic conditions are violated by the rheological model, they may, but need not, be violated, by the compliance function. In order to be sure that the compliance function is correct, it is thus highly desirable, although not required that the thermodynamic conditions be satisfied by the rheological model. Thus, all springs and all dashpots composing the rheological model must satisfy the thermodynamic conditions, i.e. the dissipation (dissipated power) has to be positive in each basic rheological element. In this context, Bazant has demonstrated that two distinct constitutive laws are necessary to define the aging spring behavior (Bazant, 1979; Bazant et al., 1993). Hooke's law (7) for the softening spring behavior and the tangent law (8) for the hardening spring behavior are as follows

$$
\begin{array}{ll}
\sigma^{(p)}(t)=b^{(p)}(t) k_{\mathrm{ref}}^{(p)} \varepsilon^{(p)}(t), & \dot{b}^{(p)}(t)<0: \text { Softening } \\
\dot{\sigma}^{(p)}(t)=b^{(p)}(t) k_{\mathrm{ref}}^{(p)} \dot{\varepsilon}^{(p)}(t), & \dot{b}^{(p)}(t)>0 \text { : Hardening }
\end{array}
$$

The aging dashpot is always defined by Newton's law:

$$
\sigma^{(q)}(t)=a^{(q)}(t) \eta_{\mathrm{ref}}^{(q)} \dot{\varepsilon}^{(q)}(t)
$$




\subsubsection{Second Principle of Thermodynamics}

The thermodynamic state of the material is defined by free energy $\psi$ in terms of observable variables, i.e., elastic strain tensor $\varepsilon^{\mathrm{e}}$, temperature $T$ and a set of internal variables $\Theta^{(n)}, n \in(1,2, \ldots, N)$ such as:

$$
\psi=\psi\left(\varepsilon^{\mathrm{e}}, T, \Theta^{(1)}, \ldots, \Theta^{(N)}\right)
$$

The latter are associated with the dissipation of the mechanical work into heat related to the irreversible material behavior. The Clausius-Duhem inequality expresses the second principle of thermodynamics, which stipulates a positive dissipation $D_{\text {tot }}$.

$$
D_{\mathrm{tot}}=\sigma: \dot{\varepsilon}^{\mathrm{an}}-\sum_{1}^{N} A^{(n)} \dot{\Theta}^{(n)}-\vec{q} \cdot \frac{\vec{\nabla} T}{T} \geq 0
$$

$\varepsilon^{\text {an }}=\varepsilon-\varepsilon^{\mathrm{e}}$ represents the anelastic strain and $A^{(n)}$ the thermodynamic force associated with the internal variable $\Theta^{(n)}$.

\subsubsection{Thermodynamic Restrictions for Aging Spring}

The aging spring behavior is associated with the modulus variation over time. Aging involves dissipation of a part of the mechanical energy provided by the spring. In the formulation of the Helmholtz specific free energy noted $\psi$, we take the tensor of elastic strain $\varepsilon^{\mathrm{e}}$ and the temperature $T$ as state variables, and the aging parameter $b(t)$ as an internal variable, such as $\psi=\psi\left(\varepsilon^{\mathrm{e}}, T, b\right)$. For an aging spring, the free energy $\psi$ is always defined by the relationship:

$$
\psi=\frac{1}{2 \rho} b(t) k_{\mathrm{ref}}\left(\varepsilon^{e}(t)\right)^{2}
$$

where, $k_{\text {ref }}$ is the modulus reference. The intrinsic dissipation is deduced from the Clausius-Duhem inequality by considering only one internal variable $b$ associated with the thermodynamic force $A$, such as:

$$
D_{\mathrm{tot}}=\sigma: \dot{\varepsilon}^{\mathrm{an}}-A \dot{b}-\vec{q} \frac{\vec{\nabla} T}{T} \geq 0
$$

with the following state equations:

$$
\sigma=\rho \frac{\partial \psi}{\partial \varepsilon^{e}}, \quad s=-\frac{\partial \psi}{\partial T} \quad \text { and } \quad A=\rho \frac{\partial \psi}{\partial b}
$$


Softening spring behavior: The softening spring behavior is defined by Hooke's law:

$$
\varepsilon(t)=\frac{\sigma(t)}{b(t) k_{\mathrm{ref}}} \quad \text { with } \quad \varepsilon(t)=\varepsilon^{\mathrm{e}}(t)+\varepsilon^{\mathrm{an}}(t)
$$

By definition, the elastic strain $\varepsilon^{\mathrm{e}}$ is defined as the strain increment observed during an instantaneous unloading (at this time the climatic conditions are constant). Thus, the elastic strain $\varepsilon^{\mathrm{e}}$ will always be defined by the following relationship:

$$
\varepsilon^{\mathrm{e}}(t)=\frac{\sigma}{b(t) k_{\mathrm{ref}}} \quad \text { and } \quad \varepsilon^{\mathrm{e}}(t)=\frac{\dot{\sigma}(t)}{b(t) k_{\mathrm{ref}}}-\frac{\dot{b}(t) \sigma(t)}{b^{2}(t) k_{\mathrm{ref}}}
$$

The anelastic strain $\varepsilon^{\text {an }}$ is deduced from relationships (15) and (16):

$$
\varepsilon^{\mathrm{an}}(t)=\varepsilon(t)-\varepsilon^{\mathrm{e}}(t)=0
$$

From the experession of the free energy (12) and the expression (14), the thermodynamic force $A$ associated with the internal variable $b$ is defined by:

$$
A=\frac{1}{2} k_{\mathrm{ref}}\left(\varepsilon^{\mathrm{e}}(t)\right)^{2}=\frac{\sigma^{2}(t)}{2 b^{2}(t) k_{\mathrm{ref}}}
$$

By substituting the relationships (17) and (18) in the Clausius-Duhem inequality (13) and supposing a homogeneous variation of the temperature $(\vec{\nabla} T=0)$, the total dissipation from (13) can be simplified by:

$$
D_{k_{S}}=-\frac{\dot{b}(t) \sigma^{2}(t)}{2 b^{2}(t) k_{\mathrm{ref}}} \geq 0
$$

When spring modulus depends on time, $k(t)=b(t) k_{\text {ref }}, k_{\text {ref }} \geq 0$, the inequality (19) imposes:

$$
\dot{b}(t) \leq 0
$$

Finally, the inequality (20) demonstrates that the Hooke's law can be used for softening behavior. In this case, it is necessary to employ an adapted law for hardening behavior.

Hardening spring behavior: The constitutive law of the hardening spring behavior must be represented by the tangent law proposed by Bazant (1979):

$$
\dot{\varepsilon}(t)=\frac{\dot{\sigma}(t)}{b(t) k_{\mathrm{ref}}} \quad \text { with } \quad \dot{\varepsilon}(t)=\dot{\varepsilon}^{\mathrm{e}}(t)+\dot{\varepsilon}^{\mathrm{an}}(t)
$$


As previously, the elastic strain $\varepsilon^{\mathrm{e}}$ is obtained during an instantaneous unloading and always defined by the relationship:

$$
\varepsilon^{\mathrm{e}}(t)=\frac{\sigma}{b(t) k_{\mathrm{ref}}} \quad \text { with } \quad \dot{\varepsilon}^{\mathrm{e}}(t)=\frac{\dot{\sigma}(t)}{b(t) k_{\mathrm{ref}}}+\frac{\dot{b}(t) \sigma(t)}{b^{2}(t) k_{\mathrm{ref}}}
$$

Considering the relationships (21) and (22), the anelastic strain rate $\dot{\varepsilon}^{\text {an }}$ can be expressed by:

$$
\dot{\varepsilon}^{\mathrm{an}}(t)=\dot{\varepsilon}(t)-\dot{\varepsilon}^{\mathrm{e}}(t)=\frac{\dot{b}(t) \sigma}{b^{2}(t) k_{\mathrm{ref}}}
$$

In a softening spring assumption, the variable $A$ is deduced from the free energy (12) and the relationship (14):

$$
A=\rho \frac{\partial \psi}{\partial b}=\frac{1}{2} k_{\mathrm{ref}}\left(\varepsilon^{\mathrm{e}}(t)\right)^{2}=\frac{\sigma^{2}(t)}{2 b^{2}(t) k_{\mathrm{ref}}}
$$

According to the definition of $A$ for softening and hardening behavior, Equations (18) and (24), respectively, we can conclude that a positive or negative variation of moisture content, in a constant loading configuration, has the same effect about the free energy variation. The most important effect is the movement of link water molecules.

By introducing expressions (23) and (24) in the Clausius-Duhem inequality (13) and assuming a homogeneous temperature variation $(\vec{\nabla} T=0)$, we obtain the following total dissipation for a hardening spring behavior:

$$
D_{k_{H}}=\sigma: \dot{\varepsilon}^{\mathrm{an}}-A \dot{b}=\frac{\dot{b}(t) \sigma^{2}(t)}{2 b^{2}(t) k_{\mathrm{ref}}} \geq 0
$$

In this case, when the spring modulus varies over time, $k(t)=b(t) k_{\text {ref }}$ and $k_{\text {ref }} \geq 0$, the inequality (25) is checked if:

$$
\dot{b}(t) \geq 0
$$

The spring modulus $k$ is always positive and the use of the tangent law is only validated for a hardening spring behavior. We remark that the dissipation for an aging spring is given by:

$$
D_{k}=\frac{|\dot{b}(t)| \sigma^{2}(t)}{2 b^{2}(t) k_{\mathrm{ref}}} \geq 0
$$




\subsubsection{Thermodynamic Restrictions for Aging Dashpot}

The aging dashpot behavior is translated by the viscosity variation over time and it is always defined by Newton's law (67):

$$
\sigma(t)=a(t) \eta_{\mathrm{ref}} \dot{\varepsilon}(t) \quad \text { and } \quad \dot{\varepsilon}(t)=\dot{\varepsilon}^{\mathrm{e}}(t)+\dot{\varepsilon}^{\mathrm{an}}(t)
$$

The free energy and the elastic strain are zero $\left(\psi=0, \varepsilon^{\mathrm{e}}=0\right)$. The relationship (67) is then written as:

$$
\dot{\sigma}(t)=a(t) \eta_{\mathrm{ref}} \dot{\varepsilon}^{\mathrm{an}}(t)
$$

Moreover, if the free energy is zero, the thermodynamic force associated with $b$ is also zero $(A=0)$. For transformation at a homogeneous temperature, the Clausius-Duhem inequality (68) is reduced to:

$$
D_{\eta}=\sigma: \dot{\varepsilon}^{\mathrm{an}}=\frac{\sigma^{2}(t)}{a(t) \eta_{\mathrm{ref}}} \geq 0
$$

If we assume that dashpot viscosity is constant $(\dot{a}(t)=0)$ and taken as reference $\left(\eta_{\mathrm{ref}}, a=1\right)$, the Clausius-Duhem inequality (30) is validated if:

$$
\eta_{\text {ref }} \geq 0 \quad \forall \quad w_{\text {ref }}
$$

When dashpot viscosity varies over time, $\eta(t)=a(t) \eta_{\text {ref }}$ and $\eta_{\text {ref }} \geq 0$, the inequality (30) induces:

$$
a(t) \geq 0
$$

In conclusion, the reference viscosity $\eta_{\text {ref }}$ is always positive and Newton's law is always satisfied thermodynamically if the dashpot viscosity is positive.

\subsection{Aging Viscoelastic Behavior}

\subsubsection{Constitutive Law for Softening Viscoelastic Material}

This section deals with an aging rheological viscoelastic model whose spring stiffness decreases over time (increase of moisture content). In order to develop the corresponding integral formulation, it is necessary to introduce the basic laws (9) and (7) for each spring and each dashpot composing the rheological model. According to the law (7), the instantaneous elastic strain is expressed in the following form:

$$
\varepsilon^{(0)}(t)=\frac{1}{k_{\mathrm{ref}}^{(0)}} \frac{\sigma(t)}{b^{(0)}(t)}
$$




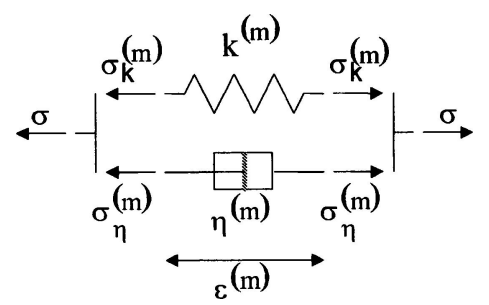

Figure 2. Stress decomposition.

We can introduce the derivation form of the stress expression as follows:

$$
\sigma(t)=\int_{0^{-}}^{t} \frac{\partial \sigma(\tau)}{\partial \tau} d \tau
$$

Introducing the form (34) in the Equation (33), we obtain ( $t$ and $\tau$ are independent variables):

$$
\varepsilon^{(0)}(t)=\int_{0^{-}}^{t} J_{a}^{(0)}(t, \tau) \frac{\partial \sigma(\tau)}{\partial \tau} d \tau \quad \text { with } \quad J_{a}^{(0)}(t, \tau)=\frac{1}{b^{(0)}(t) k_{\mathrm{ref}}^{(0)}}
$$

The strain expression of each Kelvin-Voigt cell is deduced from the stress decomposition (36) in Figure 2.

$$
\sigma(t)=\sigma_{k}^{(m)}(t)+\sigma_{\eta}^{(m)}(t)
$$

By substituting the relationships (9) and (7) in the Equation (36), we obtain the following differential equation:

$$
\dot{\varepsilon}^{(m)}+\left(\frac{b^{(m)}}{a^{(m)}} \lambda_{\text {ref }}^{(m)}\right) \varepsilon^{(m)}=\frac{\sigma}{a^{(m)} \eta_{\text {ref }}^{(m)}} \quad \text { with } \quad \lambda_{\text {ref }}^{(m)}=\frac{k_{\text {ref }}^{(m)}}{\eta_{\text {ref }}^{(m)}}
$$

The resolution of the Equation (37) enables us to calculate the strain of each Kelvin-Voigt cell:

$$
\varepsilon^{(m)}(t)=\int_{0^{-}}^{t} J_{a}^{(m)}(t, \tau) \frac{\partial \sigma(\tau)}{\partial \tau} d \tau,
$$

with

$$
J_{a}^{(m)}(t, \tau)=\int_{\tau}^{t} \frac{e^{-\lambda_{\mathrm{ref}}^{(m)} \int_{\vartheta}^{t} \frac{b^{(m)}(\mu)}{a^{(m)}(\mu)} d \mu}}{a^{(m)}(\vartheta) \eta_{\mathrm{ref}}^{(m)}} d \vartheta
$$




\subsubsection{Constitutive Law for Hardening Viscoelastic Material}

In order to take into account the spring modulus which increases when the wood moisture content decreases, we take the decomposition of strains (2) and stresses (36). The instantaneous response is expressed by integrating the constitutive equation of the hardening spring behavior (8). This adapted form is:

$$
\frac{\partial \varepsilon^{(0)}}{\partial \tau}=\frac{1}{b^{(0)}(\tau) k_{\mathrm{ref}}^{(0)}} \frac{\partial \sigma}{\partial \tau}
$$

Integrating the expression (39), we obtain:

$$
\varepsilon^{(0)}(t)=\int_{0^{-}}^{t} J_{r}^{(0)}(t, \tau) \frac{\partial \sigma(\tau)}{\partial \tau} d \tau \quad \text { with } \quad J_{r}^{(0)}(t, \tau)=\frac{1}{b^{(0)}(\tau) k_{\mathrm{ref}}^{(0)}}
$$

By comparing the expressions (35) and (40), we can observe that the softening law integrates the past history of stresses and the hardening law must integrate in the same time the past history of material properties.

The strain calculation of each Kelvin cell is also carried out by substituting the basic laws (9) and (8) in (36). We thus obtain a second-order differential equation such as:

$$
\ddot{\varepsilon}^{(m)}(t)+\left(\frac{b^{(m)}(t)}{a^{(m)}(t)} \lambda_{\mathrm{ref}}^{(m)}+\frac{\dot{a}^{(m)}(t)}{a^{(m)}(t)}\right) \dot{\varepsilon}^{(m)}(t)=\frac{\dot{\sigma}(t)}{a^{(m)}(t) \eta_{\mathrm{ref}}^{(m)}}
$$

The resolution of the Equation (41) is carried out in two steps. A first integration allows us to define the following rate of strain:

$$
\dot{\varepsilon}^{(m)}(t)=\int_{0^{-}}^{t} \frac{e^{-\lambda_{\mathrm{ref}}^{(m)} \int_{\tau}^{t} \frac{b^{(m)}(\mu)}{a^{(m)}(\mu)} d \mu}}{a^{(m)}(t) \eta_{\mathrm{ref}}^{(m)}} \frac{\partial \sigma(\tau)}{\partial \tau} d \tau
$$

A second integration enables us to obtain the strain $\varepsilon^{(m)}$, such as:

$$
\varepsilon^{(m)}(t)=\int_{\vartheta=0}^{\vartheta=t}\left(\int_{\tau=0}^{\tau=\vartheta} \frac{e^{-\lambda_{\mathrm{ref}}^{(m)} \int_{\tau}^{\vartheta} \frac{b^{(m)}(\mu)}{a^{(m)}(\mu)} d \mu}}{a^{(m)}(\vartheta) \eta_{\mathrm{ref}}^{(m)}} \frac{\partial \sigma(\tau)}{\partial \tau} d \tau\right) d \vartheta
$$

By substituting the integration order, the integral (43) with $\vartheta \in[0, t]$ and $\tau \in[0, \vartheta]$ is the same as the integral (44) with $\tau \in[0, t]$ and $\vartheta \in[\tau, t]$ :

$$
\varepsilon^{(m)}(t)=\int_{\tau=0}^{\tau=t}\left(\int_{\vartheta=\tau}^{\vartheta=t} \frac{e^{-\lambda_{\mathrm{ref}}^{(m)} \int_{\tau}^{\vartheta} \frac{b^{(m)}(\mu)}{a^{(m)}(\mu)} d \mu}}{a^{(m)}(\vartheta) \eta_{\mathrm{ref}}^{(m)}} d \vartheta\right) \frac{\partial \sigma(\tau)}{\partial \tau} d \tau
$$


The compliance function $J_{\tau}^{(m)}(t, \tau)$ is deduced from the relationship (44), such as:

with

$$
\varepsilon^{(m)}(t)=\int_{0^{-}}^{t} J_{r}^{(m)}(t, \tau) \frac{\partial \sigma(\tau)}{\partial \tau} d \tau,
$$

$$
J_{r}^{(m)}(t, \tau)=\int_{\tau}^{t} \frac{e^{-\lambda_{\mathrm{ref}}^{(m)} \int_{\tau}^{\vartheta} \frac{b^{(m)}(\mu)}{a^{(m)}(\mu)} d \mu}}{a^{(m)}(\vartheta) \eta_{\mathrm{ref}}^{(m)}} d \vartheta
$$

Finally, the comparison of strain expressions (38) and (45) shows us the differences for the past history integration of stresses and material properties for a softening and a hardening law. However, the main difficulty is the coupling between these two behaviors in variable moisture content conditions.

\subsection{Moisture-Induced Shrinkage-Swelling}

In the hygroscopic field, an increase and decrease of moisture content generates a swelling and shrinkage, respectively. If this phenomenon is observed without mechanical loading through the free strains, this is modified when the structure is loaded (Hunt, 1984, 1988). This process highlights a coupling between the moisture variations and the mechanical state. The shrinkage-swelling strain, noted $\varepsilon^{(w)}$, is expressed by the following formulation:

with

$$
\Delta \varepsilon_{n}^{(w)}=\int_{t_{n-1}}^{t_{n}}\left[\alpha(t-\tau)+m \varepsilon^{(\mathrm{ve})}(\tau)\right] \frac{\partial w}{\partial \tau} d \tau
$$

$$
\alpha(t)=\alpha^{(0)}+\sum_{m=1}^{M} \alpha^{(m)}\left(1-e^{-\lambda_{\alpha}^{(m)}} t\right)
$$

$\alpha(t)$ is the uniaxial coefficient of free shrinkage-swelling and $m$ is the mechanosorptive coefficient showing the coupling between shrinkage-swelling and viscoelastic strain $\varepsilon^{(\mathrm{ve})}$. The coefficient $m$ also translates the difference in amplitude between the shrinkage strain and swelling strain under stress (Ranta-Maunus, 1975), such as:

$$
m= \begin{cases}m_{H} & \text { if } \quad \dot{w}(t)>0: \text { moistening } \\ m_{S} & \text { if } \quad \dot{w}(t)<0: \text { drying }\end{cases}
$$

\subsection{Integral Formulation of TOTAL Strain}

\subsubsection{Softening Material}

From the total strain decomposition (2) and the relationships (33), (38), an integral form of the total strain is deduced: 


$$
\begin{aligned}
\varepsilon(t)= & \int_{0^{-}}^{t}\left(J_{a}^{(0)}(t, \tau)+\sum_{m=1}^{M} J_{a}^{(m)}(t, \tau)\right) \frac{\partial \sigma(\tau)}{\partial \tau} d \tau \\
& +\int_{0^{-}}^{t} \alpha(t-\tau) \frac{\partial w(\tau)}{\partial \tau} d \tau+\int_{0^{-}}^{t} m \varepsilon^{(\mathrm{ve})}(\tau) \frac{\partial w(\tau)}{\partial \tau} d \tau
\end{aligned}
$$

\subsubsection{Hardening Material}

In the same way, from the relationships (2), (40) and the integration of (42), the total strain is written as follows:

$$
\begin{aligned}
\varepsilon(t)= & \int_{0^{-}}^{t}\left(J_{r}^{(0)}(t, \tau)+\sum_{m=1}^{M} J_{r}^{(m)}(t, \tau)\right) \frac{\partial \sigma(\tau)}{\partial \tau} d \tau \\
& +\int_{0^{-}}^{t} \alpha(t-\tau) \frac{\partial w(\tau)}{\partial \tau} d \tau+\int_{0^{-}}^{t} m \varepsilon^{(v e)}(\tau) \frac{\partial w(\tau)}{\partial \tau} d \tau
\end{aligned}
$$

\section{Incremental Formulation}

Some works (Zienkiewicz et al., 1968) show a direct integration of the integral formulations similar to the expression (1) in a finite element code. However, the calculation of this integral function supposes the knowledge of the total history of the various fields. For the long-term prediction of the evolution of complex structures, this method encounters a problem of computer memory. In order to overcome this difficulty, we propose an explicit incremental formulation, which enables us to calculate the evolution of the various mechanical fields in an incremental time step while carrying out an equivalent storage in fields updated to each calculation step. Between two times $t_{n-1}$ and $t_{n}$, we can express the total strain decomposition (2) in an incremental form:

$$
\Delta \varepsilon_{n}=\Delta \varepsilon_{n}^{(\mathrm{ve})}+\Delta \varepsilon_{n}^{(w)}
$$

with

$$
\Delta \varepsilon_{n}^{(\mathrm{ve})}=\Delta \varepsilon_{n}^{(0)}+\sum_{m=1}^{M} \Delta \varepsilon_{n}^{(m)} \quad \text { and } \quad \Delta \varepsilon_{n}=\varepsilon\left(t_{n}\right)-\varepsilon\left(t_{n-1}\right)
$$

Thus, the various increments of this decomposition for the hardening and softening cases are defined. The strain increment $\Delta \varepsilon_{n}^{(w)}$ is common to both formulations. 


\subsection{InCREMEntal Formulation of the Softening Viscoelastic LAW}

The instantaneous strain increment $\Delta \varepsilon_{n}^{(0)}$ is obtained by considering the derivative of the integral form (33):

$$
\frac{d \varepsilon^{(0)}}{d t}=\frac{1}{k_{\mathrm{ref}}^{(0)}} \frac{d}{d t}\left(\frac{\sigma(t)}{b^{(0)}(t)}\right)=\frac{1}{k_{\mathrm{ref}}^{(0)}} \frac{d}{d t}\left(c^{(0)}(t) \sigma(t)\right)
$$

with

$$
c^{(0)}(t)=\frac{1}{b^{(0)}(t)}
$$

By integrating the expression (50) between times $t_{n-1}$ and $t_{n}$, we deduce the relationship:

$$
\Delta \varepsilon_{n}^{(0)}=\int_{t_{n-1}}^{t_{n}} \frac{d \varepsilon^{(0)}(\tau)}{d \tau} d \tau=\frac{1}{k_{\mathrm{ref}}^{(0)}} \int_{t_{n-1}}^{t_{n}} \frac{\partial}{\partial T}\left(c^{(0)}(\tau) \sigma(\tau)\right) d \tau
$$

In order to integrate the expression (51) with acceptable precision, we suppose a linear form of $\sigma$ and $c^{(0)}$ between times $t_{n-1}$ and $t_{n}$ such as:

$$
c^{(0)}(t)=c^{(0)}\left(t_{n-1}\right)+\frac{\Delta c_{n}^{(0)}}{t_{n}-t_{n-1}}
$$

and

$$
\sigma(t)=\sigma\left(t_{n-1}\right)+\frac{\Delta \sigma_{n}}{t_{n}-t_{n-1}}
$$

Introducing relations (53) in (51), we obtain the following incremental equation:

$$
\Delta \varepsilon_{n}^{(0)}=M_{a}^{(0)} \Delta \sigma_{n}+\tilde{\varepsilon}_{a}^{(0)}
$$

with

$$
M_{a}^{(0)}=\frac{c^{(0)}\left(t_{n-1}\right)+\Delta c_{n}^{(0)}}{k_{\mathrm{ref}}^{(0)}}
$$

and

$$
\tilde{\varepsilon}_{a}^{(0)}=\frac{\Delta c_{n}^{(0)}}{k_{\mathrm{ref}}^{(0)}} \sigma\left(t_{n-1}\right)
$$


The strain increment of each Kelvin cell is obtained by assuming, in the formulation (38), the following variable change:

$$
\phi=\int_{0}^{t} \frac{b^{(m)}(\zeta)}{a^{(m)}(\zeta)} d \zeta \quad \text { and } \quad \phi-\phi^{\prime}=\int_{\tau}^{t} \frac{b^{(m)}(\zeta)}{a^{(m)}(\zeta)} d \zeta
$$

$\phi$ is the reduced time. By substituting (55) in relationship (38), we obtain:

$$
\begin{aligned}
\varepsilon^{(m)}\left(t_{n}\right)= & \int_{0}^{\phi_{n-1}^{(m)}} \frac{e^{-\lambda_{\mathrm{ref}}^{(m)}\left(\phi_{n}^{(m)}-\phi^{\prime}\right)}}{n_{\mathrm{ref}}^{(m)}} c^{(m)}\left(\phi^{\prime}\right) \sigma\left(\phi^{\prime}\right) d \phi^{\prime} \\
& +\int_{\phi_{n-1}^{(m)}}^{\phi_{n}^{(m)}} \frac{e^{-\lambda_{\mathrm{ref}}^{(m)}\left(\phi_{n}^{(m)}-\phi^{\prime}\right)}}{n_{\mathrm{ref}}^{(m)}} c^{(m)}\left(\phi^{\prime}\right) \sigma\left(\phi^{\prime}\right) d \phi^{\prime}
\end{aligned}
$$

with

$$
c^{(m)}(\tau)=\frac{1}{b^{(m)}(\tau)}
$$

The relationship (56) can be transformed while revealing the term of strain calculated at time $t_{n-1}$ :

$$
\begin{aligned}
\varepsilon^{(m)}\left(t_{n}\right)= & e^{-\lambda_{\mathrm{ref}}^{(m)} \Delta \phi_{n}^{(m)}} \varepsilon^{(m)}\left(t_{n-1}\right) \\
& +\int_{\phi_{n-1}^{(m)}}^{\phi_{n}^{(m)}} \frac{e^{-\lambda_{\mathrm{ref}}^{(m)}\left(\phi_{n}^{(m)}-\phi^{\prime}\right)}}{\eta_{\mathrm{ref}}^{(m)}} c^{(m)}\left(\phi^{\prime}\right) \sigma\left(\phi^{\prime}\right) d \phi^{\prime}
\end{aligned}
$$

Thus, the strain increment is written according to the formulations (49) and (57) by the relationship:

$$
\begin{aligned}
\Delta \varepsilon_{n}^{(m)}= & \left(e^{-\lambda_{\mathrm{ref}}^{(m)} \Delta \phi_{n}^{(m)}}-1\right) \varepsilon^{(m)}\left(t_{n-1}\right) \\
& +\int_{\phi_{n-1}^{(m)}}^{\phi_{n}^{(m)}} \frac{e^{-\lambda_{\mathrm{ref}}^{(m)}}\left(\phi_{n}^{(m)}-\phi^{\prime}\right)}{\eta_{\mathrm{ref}}^{(m)}} c^{(m)}\left(\phi^{\prime}\right) \sigma\left(\phi^{\prime}\right) d \phi^{\prime}
\end{aligned}
$$

With the hypothesis of linear form of $\sigma$ and $c^{(m)}$, see adapted expressions (52) for the reduced time interval $\left[\phi_{n-1}, \phi_{n}\right]$, the strain increment $\Delta \varepsilon_{n}^{(m)}$ is given by:

$$
\Delta \varepsilon_{n}^{(m)}=M_{a}^{(m)} \Delta \sigma_{n}+\tilde{\varepsilon}_{a}^{(m)}
$$


with

$$
\begin{aligned}
& M_{a}^{(m)}=\frac{c^{(m)}\left(t_{n-1}\right)}{k_{\mathrm{ref}}^{(m)}} B^{(m)}+\frac{\Delta c_{n}^{(m)}}{k_{\mathrm{ref}}^{(m)}} C^{(m)} \\
& \tilde{\varepsilon}_{a}^{(m)}=\sigma\left(t_{n-1}\right)\left(\frac{c^{(m)}\left(t_{n-1}\right)}{k_{\mathrm{ref}}^{(m)}} A^{(m)}+\frac{\Delta c_{n}^{(m)}}{k_{\mathrm{ref}}^{(m)}} B^{(m)}\right)-\varepsilon^{(m)}\left(t_{n-1}\right) A^{(m)}
\end{aligned}
$$

and

$$
\begin{aligned}
A^{(m)} & =1-e^{-\lambda_{\text {ref }}^{(m)}} \Delta_{n}^{(m)} \\
B^{(m)} & =1-\frac{1}{\lambda_{\text {ref }}^{(m)} \Delta \phi_{n}^{(m)}}\left(1-e^{-\lambda_{\text {ref }}^{(m)} \Delta \phi_{n}^{(m)}}\right) \\
C^{(m)} & =1-\frac{2}{\lambda_{\text {ref }}^{(m)} \Delta \phi_{n}^{(m)}}\left(1-\frac{1}{\lambda_{\text {ref }}^{(m)} \Delta \phi_{n}^{(m)}}\left(1-e^{-\lambda_{\text {ref }}^{(m)} \Delta \phi_{n}^{(m)}}\right)\right) \\
\Delta \phi_{n}^{(m)} & =\phi_{n}^{(m)}-\phi_{n-1}^{(m)}
\end{aligned}
$$

\subsection{INCREMENTAL Formulation OF THE HARDENING VISCOELASTIC LAW}

The derivative of the instantaneous strain $\varepsilon^{(0)}(t)$ is developed using the expression (40) in the following way:

$$
\frac{d \varepsilon^{(0)}}{d t}=\frac{c^{(0)}(t)}{k_{\mathrm{ref}}^{(0)}} \frac{d \sigma}{d t} \quad \text { with } \quad c^{(0)}(t)=\frac{1}{b^{(0)}(t)}
$$

The integration of the expression (62) gives the following equation:

$$
\Delta \varepsilon_{n}^{(0)}=\int_{t_{n-1}}^{t_{n}} \frac{d \varepsilon^{(0)}(\tau)}{d \tau} d \tau=\frac{1}{k_{\mathrm{ref}}^{(0)}} \int_{t_{n-1}}^{t_{n}} c^{(0)}(\tau) \frac{\partial \sigma(\tau)}{\partial \tau} d \tau
$$

Assuming the similar linear form of $\sigma$ and $c^{(0)}$ between the times $t_{n-1}$ and $t_{n}$, proposed in expressions (52), the Equation (63) is written as follows:

$$
\Delta \varepsilon_{n}^{(0)}=M_{r}^{(0)} \Delta \sigma_{n}+\tilde{\varepsilon}_{r}^{(0)} u
$$

with

$$
\begin{aligned}
M_{r}^{(0)} & =\frac{1}{k_{\mathrm{ref}}^{(0)}}\left(c^{(0)}\left(t_{n-1}\right)+\frac{\Delta c_{n}^{(0)}}{2}\right) \\
\tilde{\varepsilon}_{r}^{(0)} & =0
\end{aligned}
$$


The calculation of the strain increment of the $m$ th Kelvin-Voigt cell is carried out by developing the differential Equation (41):

$$
\Delta \varepsilon_{n}^{(m)}=\int_{t_{n-1}}^{t_{n}} \frac{\partial \varepsilon^{(m)}(\tau)}{\partial \tau} d \tau=\int_{\phi_{n-1}^{(m)}}^{\phi_{n}^{(m)}} \frac{\partial \varepsilon^{(m)}\left(\phi^{\prime}\right)}{\partial \phi^{\prime}} d \phi^{\prime}
$$

As previously observed, the notation $\phi$ is the reduced time, which is given by the Equation (55). The introduction of the reduced time into the Equation (42) allows us to write:

$$
\frac{\partial \varepsilon^{(m)}(\phi)}{\partial \phi}=\frac{c^{(m)}(\phi)}{\eta_{\mathrm{ref}}^{(m)}} \int_{0}^{\phi} e^{-\lambda_{\mathrm{ref}}^{(m)}\left(\phi-\phi^{\prime}\right)} \frac{\partial \sigma}{\partial \phi^{\prime}} d \phi^{\prime}
$$

We solve the Equation (67) and introduce it in a relationship (66); so $\Delta \varepsilon_{n}(m)$ is defined by:

$$
\begin{aligned}
\Delta \varepsilon_{n}^{(m)}= & \int_{\phi_{n-1}^{(m)}}^{\phi_{n}^{(m)}} \frac{c^{(m)}(\phi)}{\eta_{\mathrm{ref}}^{(m)}} \sigma(\phi) d \phi \\
& -\frac{\lambda_{\mathrm{ref}}^{(m)}}{\eta_{\mathrm{ref}}^{(m)}} \int_{\phi_{n-1}^{(m)}}^{\phi_{n}^{(m)}}\left(c^{(m)}(\phi) \int_{0}^{\phi} e^{-\lambda_{\mathrm{ref}}^{(m)}\left(\phi-\phi^{\prime}\right)} \sigma\left(\phi^{\prime}\right) d \phi^{\prime}\right) d \phi
\end{aligned}
$$

Assuming a linear form of $\sigma$ and $c^{(m)}$, in the reduced time interval $\left[\phi_{n-1}, \phi_{n}\right]$, we obtain:

$$
\Delta \varepsilon_{n}^{(m)}=M_{r}^{(m)} \Delta \sigma_{n}+\tilde{\varepsilon}_{r}^{(m)}
$$

with

$$
\begin{aligned}
M_{r}^{(m)}= & \frac{c^{(m)}\left(t_{n-1}\right)}{k_{\mathrm{ref}}^{(m)}} B^{(m)}+\frac{\Delta c_{n}^{(m)}}{2 k_{\mathrm{ref}}^{(m)}} E^{(m)} \\
\tilde{\varepsilon}_{r}^{(m)}= & \sigma\left(t_{n-1}\right)\left(\frac{c^{(m)}\left(t_{n-1}\right)}{k_{\mathrm{ref}}^{(m)}} A^{(m)}-\frac{\Delta c_{n}^{(m)}}{k_{\mathrm{ref}}^{(m)}} D^{(m)}\right) \\
& +\tilde{\varepsilon}^{(m)}\left(t_{n-1}\right)\left(-c^{(m)}\left(t_{n-1}\right) A^{(m)}+\Delta c_{n}^{(m)} D^{(m)}\right)
\end{aligned}
$$

and $A^{(m)}, B^{(m)}$, and $C^{(m)}$ are defined by the relationships (61),

$$
\begin{aligned}
D^{(m)} & =e^{-\lambda_{\text {ref }}^{(m)} \Delta \phi_{n}^{(m)}}\left(1+\frac{1}{\lambda_{\text {ref }}^{(m)} \Delta \phi_{n}^{(m)}}\right)-\frac{1}{\lambda_{\text {ref }}^{(m)} \Delta \phi_{n}^{(m)}} \\
E^{(m)} & =1-\frac{2}{\lambda_{\text {ref }}^{(m)} \Delta \phi_{n}^{(m)}} D^{(m)}
\end{aligned}
$$


$\tilde{\varepsilon}^{(m)}$ is the viscoelastic strain of nonaging material at the moisture reference $w_{\text {ref }}$, such as:

$$
\tilde{\varepsilon}^{(m)}(t)=\int_{0}^{t} \frac{e^{-\lambda_{\mathrm{ref}}^{(m)}(t-\tau)}}{\eta_{\mathrm{ref}}} \sigma(\tau) d \tau
$$

The strain increment $\Delta \tilde{\varepsilon}^{(m)}$ is similar to $\Delta \varepsilon_{n}^{(m)}$ with $c^{(m)}\left(t_{n-1}\right)=1$ and $\Delta c_{n}^{(m)}=0$.

$$
\Delta \bar{\varepsilon}_{n}^{(m)}=M_{r \bar{\varepsilon}}^{(m)} \Delta \sigma_{n}+\tilde{\varepsilon}_{\tau \bar{\varepsilon}}^{(m)}
$$

with

$$
\begin{aligned}
M_{\tau \bar{\varepsilon}}^{(m)} & =\frac{B(m)}{k_{\mathrm{ref}}^{(m)}} \\
\tilde{\varepsilon}_{\tau \bar{\varepsilon}}^{(m)} & =\sigma\left(t_{n-1}\right) \frac{A^{(m)}}{k_{\mathrm{ref}}^{(m)}}-\tilde{\varepsilon}\left(t_{n-1}\right) A^{(m)}
\end{aligned}
$$

\subsection{InCRemental Formulation of Shrinkage-Swelling}

The strain increment $\Delta \varepsilon_{n}^{(w)}$ of shrinkage-swelling under mechanical loading, between the times $\left[t_{n-1}, t_{n}\right.$, ] is given by the relationship:

$$
\Delta \varepsilon_{n}^{(w)}=\int_{t_{n-1}}^{t_{n}} \alpha(t-\tau) \frac{\partial w(\tau)}{\partial \tau} d \tau+\int_{t_{n-1}}^{t_{n}} m \varepsilon^{\nu e}(\tau) \frac{\partial w(\tau)}{\partial \tau} d \tau
$$

To facilitate the integration of the Equation (75), $\varepsilon^{(\mathrm{ve})}$ and $w$ are linear during the time increment and we deduce from it the following expression:

$$
\Delta \varepsilon_{n}^{(w)}=\left[M^{(w)}+m\left(\frac{\Delta \varepsilon^{(\mathrm{ve})}}{2}+\varepsilon^{(\mathrm{ve})}\left(t_{n-1}\right)\right)\right] \Delta w_{n}+\tilde{\varepsilon}^{(w)}
$$

with

$$
\begin{aligned}
M^{(w)}= & \alpha^{(0)}+\sum_{r=1}^{R} \alpha^{(r)}\left(1-\frac{1}{\lambda_{\alpha}^{(r)}}\left(1-e^{-\lambda_{\alpha}^{(r)}} \Delta t_{n}\right)\right) \\
\tilde{\varepsilon}^{(w)}= & \sum_{r=1}^{R} \alpha^{(r)}\left(1-e^{-\lambda_{\alpha}^{(r)} \Delta t_{n}}\right) w\left(t_{n-1}\right) \\
& +\sum_{r=1}^{R}\left(e^{-\lambda_{\alpha}^{(r)} \Delta t_{n}}-1\right) \varepsilon^{(w)}\left(t_{n-1}\right)
\end{aligned}
$$




\subsection{InCREMENTAL Formulation OF the TOtAl STRAin}

The total strain increment $\Delta \varepsilon_{n}$ is the sum of the viscoelastic strain increment and the shrinkage-swelling strain increment. In the case of a softening behavior, it is calculated by summation of the relationships (53), (59), and (75). At the time of the hardening phase, this increment rises from the combination of the expressions (63), (84), and (75). Finally, we obtain:

$$
\Delta \varepsilon_{n}=M \Delta \sigma_{n}+\tilde{\varepsilon}
$$

with

$$
\begin{array}{ll}
M=M_{a}, & \tilde{\varepsilon}=\tilde{\varepsilon}_{a}: \text { Softening } \\
M=M_{r}, & \tilde{\varepsilon}=\tilde{\varepsilon}_{r}: \text { Hardening }
\end{array}
$$

$M$ is the equivalent viscoelastic compliance, which makes it possible to translate the effect of the linear variation of the stress during the time increment.

$$
\begin{aligned}
& M_{a}=\left(1+\frac{m}{2}\right)\left(M_{a}^{(0)}+\sum_{m=1}^{M} M_{a}^{(m)}\right) \\
& M_{r}=\left(1+\frac{m}{2}\right)\left(M_{a}^{(0)}+\sum_{m=1}^{M} M_{a}^{(m)}\right)
\end{aligned}
$$

The environmental effect and the storage of the total history of the mechanical loading are taken into account as an initial strain noted $\tilde{\varepsilon}$.

$$
\begin{aligned}
\tilde{\varepsilon}_{a}= & \left(M^{(w)}+m \varepsilon^{(v e)}\left(t_{n-1}\right)\right) \Delta w_{n} \\
& +(1+m)\left(\tilde{\varepsilon}_{a}^{(0)}+\sum_{m=1}^{M} \tilde{\varepsilon}_{a}^{(m)}\right)+\tilde{\varepsilon}^{(w)} \\
\tilde{\varepsilon}_{r}= & \left(M^{(w)}+m \varepsilon^{(v e)}\left(t_{n-1}\right)\right) \Delta w_{n} \\
& +(1+m)\left(\tilde{\varepsilon}_{r}^{(0)}+\sum_{m=1}^{M} \tilde{\varepsilon}_{r}^{(m)}\right)+\tilde{\varepsilon}^{(w)}
\end{aligned}
$$

In the Equation (79), all the terms are given according to reduced time increment $\Delta \phi_{n}$. We assume that the function $\frac{b^{(m)}}{a^{(m)}}$ is linear in the time interval $\left[t_{n-1}, t_{n}\right]$ :

$$
\begin{aligned}
\Delta \Phi_{n}^{(m)} & =\int_{t_{n-1}}^{t_{n}} \frac{b^{(m)}(\mu)}{a^{(m)}(\mu)} d \mu \\
& =\frac{\Delta t_{n}}{2 c^{(m)}\left(t_{n}\right) a^{(m)}\left(t_{n}\right)}+\frac{\Delta t_{n}}{2 c^{(m)}\left(t_{n-1}\right) a^{(m)}\left(t_{n-1}\right)}
\end{aligned}
$$




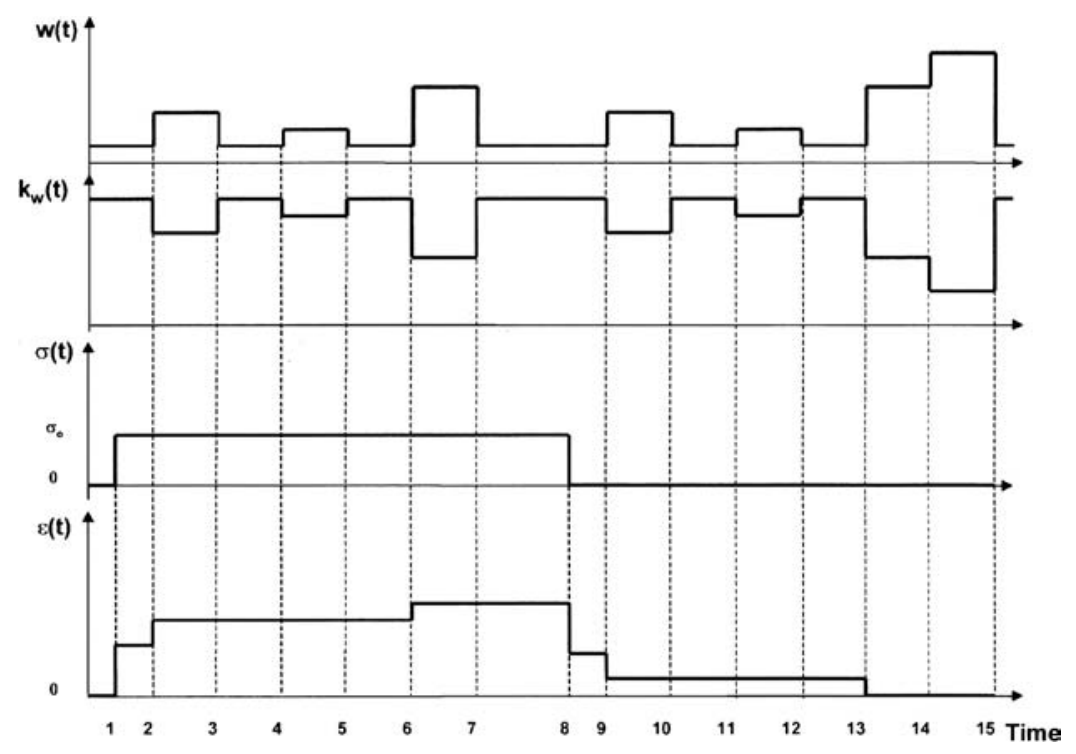

Figure 3. Elastic behavior under moisture cycles.

\subsection{Behavior Under Nonmonotonous Moisture Changes}

The incremental formulation (79) presents a similar form for softening and hardening. However, the history terms $\tilde{\varepsilon}_{a}$ and $\tilde{\varepsilon}_{r}$ are not updated in an identical way. When the formulation is used in monotonous phase, i.e., simply softening or simply hardening, the two incremental forms are employed in an uncoupled way (Randriambololona, 2001). On the other hand, if we wish to model the effects of nonmonotonous moisture evolution, we have to update the material history so that it is compatible between the end of drying (hardening) and the beginning of moistening (softening).

\subsubsection{Elastic Behavior}

For example, we take an elastic behavior modeled by a spring according to the relationships (53) and (63).

A tensile test with loading-unloading under modulus variations is represented in Figure 3. The response is calculated by using the spring constitutive laws and the superposition principle.

When material is moistened, it softens. During drying, the material hardens. Under constant load, the strain process is activated by moistening. Without mechanical load variation, the strain remains constant. The material keeps in memory the moisture history during the loading period. The strain is totally reversible if the moisture variations are the same during loading and unloading. This behavior is similar to the atypical behavior already presented by Gril (1988) with the hygro-lock model. 
In order to simulate this behavior, the storage history must be updated between the softening (80) and the hardening (78) formulations. We must consider the three following cases:

Case 1: The moisture level never before reached is now exceeded $\left(w\left(t_{n}\right) \geq\right.$ $\left.w_{\max }\right)$, the material softens, and the strain increment is defined by:

$$
\Delta \varepsilon_{x}^{(0)}=\frac{c^{(0)}\left(t_{n}\right)}{k_{\mathrm{ref}}^{(0)}} \Delta \sigma_{x}+\frac{\Delta c_{x}^{(0)}}{k_{\mathrm{ref}}^{(0)}} \sigma\left(t_{x}\right)
$$

with $t_{x}=t\left(w_{\max }\right), \Delta \varepsilon_{x}^{(0)}=\varepsilon^{(0)}\left(t_{n}\right)-\varepsilon\left(t_{x}\right), \Delta \sigma_{x}^{(0)}=\sigma^{(0)}\left(t_{n}\right)-\sigma\left(t_{x}\right), \Delta c_{x}^{(0)}=$ $c^{(0)}\left(t_{n}\right)-c\left(t_{x}\right)$

The increment of elastic strain between time $t_{n-1}$ and $t_{n}$, is written as:

$$
\begin{aligned}
\Delta \varepsilon_{n}^{(0)}= & \frac{c^{(0)}\left(t_{n}\right)}{k_{\mathrm{ref}}^{(0)}} \Delta \sigma_{n}+\frac{c^{(0)}\left(t_{n}\right)}{k_{\mathrm{ref}}^{(0)}}\left(\sigma\left(t_{n-1}\right)-\sigma\left(t_{x}\right)\right) \\
& +\frac{\Delta c_{x}^{(0)}}{k_{\mathrm{ref}}^{(0)}} \sigma\left(t_{x}\right)+\varepsilon^{(0)}\left(t_{x}\right)-\varepsilon^{(0)}\left(t_{n-1}\right)
\end{aligned}
$$

Case 2: The moisture content is higher than the moisture content reached at the last moistening, but lower than the maximal moisture content $\left(w_{\max } \geq w\left(t_{n}\right) \geq w_{h}\right)$. The elastic strain is updated as follows:

$$
\Delta \varepsilon_{x}^{(0)}=\frac{c^{(0)}\left(t_{n}\right)}{k_{\mathrm{ref}}^{(0)}} \Delta \sigma_{x}
$$

The strain increment $\Delta \varepsilon_{n}^{(0)}$ is given by this relationship:

$$
\Delta \varepsilon_{n}^{(0)}=\frac{c^{(0)}\left(t_{n}\right)}{k_{\mathrm{ref}}^{(0)}} \Delta \sigma_{n}+\frac{c^{(0)}\left(t_{n}\right)}{k_{\mathrm{ref}}^{(0)}}\left(\sigma_{(n-1)}-\sigma\left(t_{x}\right)\right)+\varepsilon^{(0)}\left(t_{x}\right)-\varepsilon_{\left(t_{n-1}\right)}^{(0)}
$$

Case 3: The moisture content is lower than the moisture content at the last moistening $w_{h} \geq w\left(t_{n}\right)$. The strain is updated as follows:

$$
\Delta \varepsilon_{h}^{(0)}=\frac{C^{(0)}\left(t_{n}\right)}{k_{\mathrm{ref}}^{(0)}} \Delta \sigma_{h}
$$

with $t_{h}=t\left(w_{h}\right), \Delta c_{h}^{(0)}=c^{(0)}\left(t_{n}\right)-c\left(t_{h}\right), \Delta \alpha_{h}^{(0)}=\sigma^{(0)}\left(t_{n}\right)-\sigma\left(t_{h}\right), \Delta \varepsilon_{h}^{(0)}=$ $\varepsilon^{(0)}\left(t_{n}\right)-\varepsilon\left(t_{h}\right)$ 
We obtain the increment of elastic strain $\Delta \varepsilon_{n}$ from the relationship (90), such as:

$$
\Delta \varepsilon_{n}^{(0)}=\frac{c^{(0)}\left(t_{n}\right)}{k_{\mathrm{ref}}^{(0)}} \Delta \sigma_{n}+\frac{c^{(0)}\left(t_{n}\right)}{k_{\mathrm{ref}}^{(0)}}\left(\sigma_{n-1}-\sigma\left(t_{h}\right)\right)+\varepsilon^{(0)}\left(t_{h}\right)-\varepsilon^{(0)}\left(t_{n-1}\right)
$$

\subsubsection{Viscoelastic Behavior}

For the viscoelastic behavior, we use the same update as on elastic spring, on the Kelvin-Voigt cells.

Case 1: The material softens and the viscoelastic strain is updated between time $t_{x}$ and $t_{n}$, as follows:

$$
\Delta \varepsilon_{x}^{(m)}=M_{a x}^{(m)} \Delta \sigma_{x}+\tilde{\varepsilon}_{a x}^{(m)}
$$

with

$$
\begin{aligned}
& M_{a x}^{(m)}=\frac{c^{(m)}\left(t_{x}\right)}{k_{\mathrm{ref}}^{(m)}} B_{x}^{(m)}+\frac{\Delta c_{x}^{(m)}}{k_{\mathrm{ref}}^{(m)}} C_{x}^{(m)} \\
& \tilde{\varepsilon}_{a x}^{(m)}=\sigma\left(t_{x}\right)\left(\frac{c^{(m)}\left(t_{x}\right)}{k_{\mathrm{ref}}^{(m)}} A_{x}^{(m)}+\frac{\Delta c_{x}^{(m)}}{k_{\mathrm{ref}}^{(m)}} B_{x}^{(m)}\right)-\varepsilon^{(m)}\left(t_{x}\right) A_{x}^{(m)}
\end{aligned}
$$

and

$$
\begin{aligned}
A_{x}^{(m)} & =1-e^{-\lambda_{\mathrm{ref}}^{(m)} \Delta \phi_{x}^{(m)}} \\
B_{x}^{(m)} & =1-\frac{1}{\lambda_{\mathrm{ref}}^{(m)} \Delta \phi_{x}^{(m)}}\left(1-e^{-\lambda_{\mathrm{ref}}^{(m)} \Delta \phi_{x}^{(())}}\right) \\
C_{x}^{(m)} & =1-\frac{2}{\lambda_{\mathrm{ref}}^{(m)} \Delta \phi_{x}^{(m)}}\left(1-\frac{1}{\lambda_{\mathrm{ref}}^{(m)} \Delta \phi_{x}^{(m)}}\left(1-e^{-\lambda_{\mathrm{ref}}^{(m)} \Delta \phi_{x}^{(m)}}\right)\right) \\
\Delta \phi_{x}^{(m)} & =\phi_{n}^{(m)}-\phi_{x}^{(m)}
\end{aligned}
$$

Case 2: The increment of viscoelastic strain, between time $t_{x}$ and $t_{n}$, is given by the following relationship:

$$
\Delta \varepsilon_{x}^{(m)}=M_{r x}^{(m)} \Delta \sigma_{x}+\tilde{\varepsilon}_{r x}^{(m)}
$$

with

$$
M_{r x}^{(M)}=\frac{c^{(m)}\left(t_{x}\right)}{k_{\mathrm{ref}}^{(m)}} B_{x}^{(m)}+\frac{\Delta c_{x}^{(m)}}{2 k_{\mathrm{ref}}^{(m)}}\left(1+\frac{2}{\lambda_{\mathrm{ref}}^{(m)} \Delta \phi_{x}^{(m)}} D_{x}^{(m)}\right)
$$




$$
\begin{aligned}
\tilde{\varepsilon}_{r x}^{(m)}= & \sigma\left(t_{x}\right)\left(\frac{c^{(m)}\left(t_{x}\right)}{k_{\mathrm{ref}}^{(m)}} A_{x}^{(m)}+\frac{\Delta c_{x}^{(m)}}{k_{\mathrm{ref}}^{(m)}} B_{x}^{(m)}\right) \\
& +\bar{\varepsilon}^{(m)}\left(t_{x}\right)\left(-c^{(m)}\left(t_{x}\right) A_{x}^{(m)}+\Delta c_{x}^{(m)} c_{x}^{(m)}\right)
\end{aligned}
$$

$A_{x}^{(m)}, B_{x}^{(m)}$, and $C_{x}^{(m)}$ are defined as in the case 1 (94) and,

$$
D_{x}^{(m)}=e^{-\lambda_{\text {ref }}^{(m)} \Delta \phi_{x}^{(m)}}\left(1+\frac{1}{\lambda_{\text {ref }}^{(m)} \Delta \phi_{x}^{(m)}}\right)-\frac{1}{\lambda_{\text {ref }}^{(m)} \Delta \phi_{x}^{(m)}}
$$

Case 3: The viscoelastic strain is updated with regard to the various parameters at time $t_{h}$, such as:

$$
\Delta \varepsilon_{h}^{(m)}=M_{r h}^{(m)} \Delta \sigma_{h}+\tilde{\varepsilon}_{r h}^{(m)}
$$

with

$$
\begin{aligned}
M_{r h}^{(m)}= & \frac{c^{(m)}\left(t_{h}\right)}{k_{\mathrm{ref}}^{(m)}} B_{h}^{(m)}+\frac{\Delta c_{h}^{(m)}}{2 k_{\mathrm{ref}}^{(m)}}\left(1+\frac{2}{\lambda_{\mathrm{ref}}^{(m)} \Delta \phi_{h}^{(m)}} D_{h}^{(m)}\right) \\
\tilde{\varepsilon}_{r h}^{(m)}= & \sigma\left(t_{h}\right)\left(\frac{c^{(m)}\left(t_{h}\right)}{k_{\mathrm{ref}}^{(m)}} A_{h}^{(m)}+\frac{\Delta c_{x}^{(m)}}{k_{\mathrm{ref}}^{(m)}} B_{h}^{(m)}\right) \\
& +\bar{\varepsilon}^{(m)}\left(t_{h}\right)\left(-c^{(m)}\left(t_{h}\right) A_{h}^{(m)}+\Delta c_{h}^{(m)} C_{h}^{(m)}\right)
\end{aligned}
$$

The expressions of the functions $A_{h}^{(m)}, B_{h}^{(m)}, C_{h}^{(m)}$, and $D_{h}^{(m)}$ are the same as the functions $A_{x}^{(m)}, B_{x}^{(m)}, C_{x}^{(m)}$, and $D_{x}^{(m)}$ by substituting $\Delta \phi_{x}^{(m)}$ by $\Delta \phi_{h}^{(m)}$.

\section{Resolution by the Finite Element Method}

\subsection{Three-Dimensional Behavior}

In order to generalize the uniaxial law as a three-dimensional law, we apply the superposition principle by taking into account linear behavior. We define the strain $\pi_{i j k l}$ in the direction $i j$ generated by uniaxial stress $\sigma_{k l}$. The strain increment $\Delta \pi_{i j k l}$ is defined by:

$$
\Delta \pi_{i j k l}=M_{i j k l} \Delta \sigma_{k l}+\tilde{\pi}_{i j k l}
$$

(without summation of $k$ and $l$ ) 
We can write the strain tensor component $\varepsilon_{i j}=\sum_{k, l} \pi_{i j k l}$. We deduce from it the strain increment:

$$
\Delta \varepsilon_{i j}=\sum_{k, l} \Delta \pi_{i j k l}
$$

Thus,

$$
\Delta \varepsilon_{i j}=\sum_{k, l} M_{i j k l} \Delta \sigma_{k l}+\sum_{k, l} \tilde{\pi}_{i j k l}
$$

The formulation (102) can also take the following tensorial form:

$$
\Delta \underline{\underline{\varepsilon}}=\mathbf{M} \Delta \underline{\underline{\sigma}}+\underline{\underline{\tilde{\varepsilon}}}
$$

\subsection{Finite Element Formulation}

In order to solve the incremental equation (103) by a finite element algorithm, we employ the method proposed by Ghazlan et al. (1995), which is derived from the virtual work principle. If the nodal displacement vector increment is noted $\left\{\Delta U\left(t_{n}\right)\right\}$, the balance equation, in the discretized domain $V$, can be written as follows:

$$
\mathbf{K}_{\mathrm{T}}\left\{\Delta U\left(t_{n}\right)\right\}=\left\{\Delta F^{\mathrm{ext}}\left(t_{n}\right)\right\}+\left\{F^{\text {hist }}\left(t_{n-1}\right)\right\}
$$

$\mathbf{K}_{\mathrm{T}}$ is an equivalent tangent matrix assembled from the tensor $\mathbf{M}$ and the straindisplacement matrix $\mathbf{B}$.

$$
\mathbf{K}_{\mathrm{T}}=\int_{V} \mathbf{B}^{\mathrm{T}} \mathbf{M}^{-1} \mathbf{B} d V
$$

$\left\{\Delta F^{\mathrm{ext}}\left(t_{n}\right)\right\}$ is the exterior nodal force vector increment during the time intrement $\Delta t_{n}$.

$$
\left\{\Delta F^{\mathrm{ext}}\left(t_{n}\right)\right\}=\int_{V} \mathbf{N}^{\mathrm{T}}\left\{\Delta f_{V}\right\} d V+\int_{S_{F}} \mathbf{N}^{\mathrm{T}}\left\{\Delta f_{S}\right\} d S
$$

$N(x, y)$ is the nodal functions matrix, which connects the vector $\left\{\Delta u\left(t_{n}\right)\right\}$ displacement field increment within an element $V$, with the vector $\left\{\Delta U\left(t_{n}\right)\right\}$, so that:

$$
\left\{\Delta u\left(x, y, t_{n}\right)\right\}=\mathbf{N}(x, y)\left\{\Delta U\left(t_{n}\right)\right\}
$$




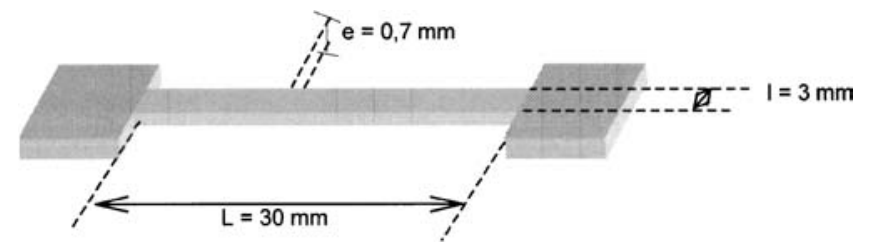

Figure 4. Clear specimen of maritime pine for tension test.

$\left\{F^{\text {hist }}\left(t_{n-1}\right)\right\}$ is a pseudo-load vector which allows us to take into account the past history of the mechanical loading and the moisture variations. It is given by the following relationship:

$$
\left\{F^{\text {hist }}\left(t_{n-1}\right)\right\}=\int_{V} \mathbf{B}^{\mathrm{T}} \mathbf{M}^{-1}\left\{\tilde{\varepsilon}\left(t_{n-1}\right)\right\} d V
$$

where $\{\tilde{\varepsilon}\}$ is the vector obtained from the tensor $\underline{\underline{\varepsilon}}$ defined in the Gauss points of the elements. The balance Equation (104) is then established in the finite element program CAST3M.

\section{Application}

A great number of numerical validations were carried out in the case of monotonous evolutions of material moisture (Randriambololona, 2001). To complete this validation, we have to assume cycles of drying and moistening. We refer to the uniaxial creep-recovery tests realized by Pittet (1996) on a small clear specimen of maritime pine $l=30 \mathrm{~mm}, l=3 \mathrm{~mm}$, and $e=0.7 \mathrm{~mm}$. Tension in the longitudinal direction is performed, see Figure 4.

\subsection{Creep and Recovery Test on an Initially Dry Specimen}

The specimen is subjected to a constant tension of $18 \mathrm{MPa}$. Loading is carried out in a dry state. Two cycles of moisture are realized during the first $14 \mathrm{hr}$ and four others after unloading, as shown in Figure 5. The small size of the specimen enables us to assume an homogenous moisture content and an instantaneous balance with climate conditions. For variations of relative humidity ranging between 30 and $75 \%$, the moisture content in the specimen is from 4 to $7.62 \%$ under a constant temperature of $21.5^{\circ} \mathrm{C}$.

To decrease the number of parameters, we limit this uniaxial identification in the longitudinal direction (loading direction). During the initial creep phase in a dry reference state $\left(w_{\text {ref }}=4 \%\right)$ and in a wet reference state $\left(w_{75}=7.62 \%\right)$, the interpolation of the creep function is obtain by using a method of spectrum decomposition which proposes three Kelvin cells. Table I gives the modulus value 


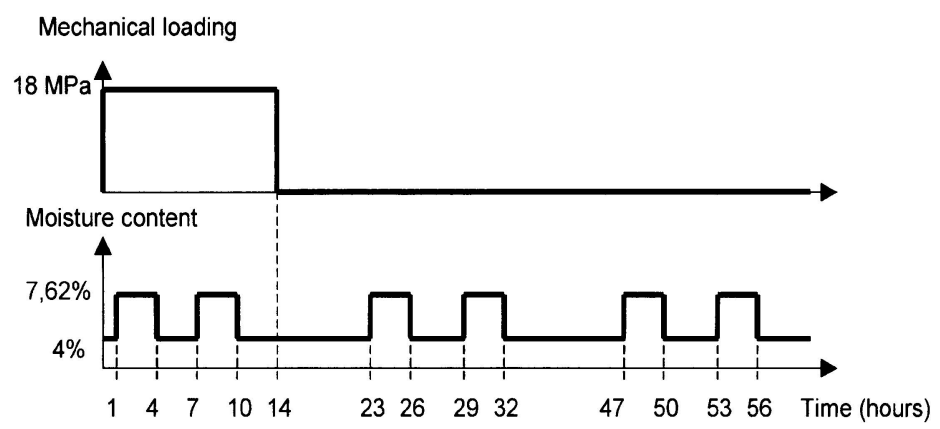

Figure 5. Mechanosorptive test.

$k_{\text {ref }}^{(p)}(\mathrm{MPa})$ and the viscosity value $\eta_{\mathrm{ref}}^{(q)}(\mathrm{MPa})$. In these conditions, the function of correction according to the moisture content, $b^{(p)}$ and $a^{(q)}$, can be defined by imposing a linear evolution versus moisture content between the two levels.

Shrinkage and swelling strain, obtained before and during mechanical loading, allow us to determine the coefficient of free shrinkage-swelling in longitudinal direction $\alpha=0,027 \%$ and the parameters of coupling in viscoelastic strain $m_{H}=$ $-0,1 \times 10^{-2}$ and $m_{S}=-3,4 \times 10^{-2}$.

Figure 6 shows that the procedure gives numerical results close to experimental results. Simulation exhibits the characteristic effect of the first moistening under load, which generates a great increase in strains; an effect that one does not find with the second moistening. After unloading, under a low moisture content, residual strains appear, a part of which will be covered during the first moistening. The residual strains at the end of the test are obtained from the accumulation of the nonrecoverable strains due to $m_{S}$ with each phase of drying. We observe an excellent simulation of behavior during the recovery period. Finally, numerical calculation allows for a decoupling of the various effects (viscoelasticity, free shrinkage-swelling, and stress influence on shrinkage-swelling) which we could not realize directly by the experimental results.

Table I. Parameters of the rheological model

\begin{tabular}{ll}
\hline Reference state $w_{\text {ref }}=4 \%$ & Function of correction \\
\hline$k_{\text {ref }}^{(0)}=9.5 \times 10^{3}$ & $b^{(0)}=1-0.066 \times(w-4)$ \\
$k_{\text {ref }}^{(1)}=8.6 \times 10^{5}$ & $b^{(1)}=1-0.26 \times(w-4)$ \\
$k_{\text {ref }}^{(2)}=3.8 \times 10^{4}$ & $b^{(2)}=1-0.38 \times(w-4)$ \\
$k_{\text {ref }}^{(3)}=5.3 \times 10^{4}$ & $b^{(3)}=1-0.131 \times(w-4)$ \\
$\eta_{\text {ref }}^{(1)}=6.1 \times 10^{8}$ & $a^{(1)}=1-0.264 \times(w-4)$ \\
$\eta_{\text {ref }}^{(2)}=2.7 \times 10^{8}$ & $a^{(2)}=1-0.079 \times(w-4)$ \\
$\eta_{\text {ref }}^{(3)}=3.7 \times 10^{8}$ & $a^{(3)}=1-0.16 \times(w-4)$ \\
\hline
\end{tabular}




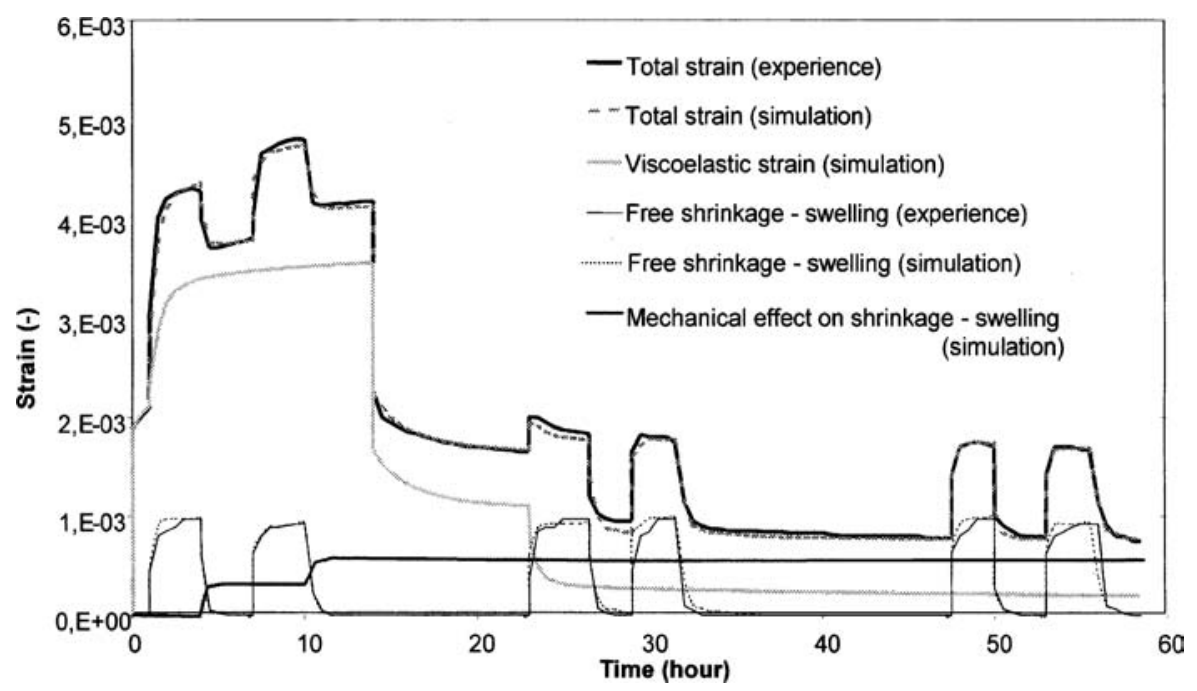

Figure 6. Results of the simulation.

\subsection{Creep and Recovery Test on an Initially Wet Specimen}

The same type of specimen is subjected to a tension loading of $32 \mathrm{MPa}$ maintained for $17 \mathrm{hr}$. Loading is carried out in a wet state. Three cycles of moisture are produced during the creep phase and three others during the recovery phase, as shown in Figure 7. For variations of relative humidity between 30 and $75 \%$, the moisture content in the specimen varies between $4 \%$ and $7.62 \%$ under a constant temperature $T=21.5^{\circ} \mathrm{C}$.

Shrinkage and swelling strain, obtained before and during mechanical loading, enables us to obtain the coefficient of free longitudinal shrinkage and swelling $\alpha=0.017 \%$ and the factors describing the influence of the mechanical state on the process of shrinkage-swelling $m_{H}=-3 \times 10^{-2}$ and $m_{S}=-6,5 \times 10^{-2}$.

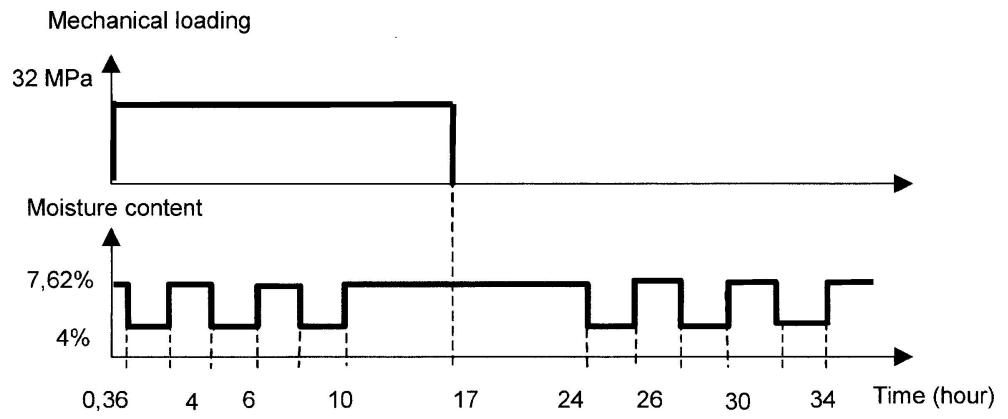

Figure 7. Mechanosorptive test. 
Table II. Parameters of the rheological model

\begin{tabular}{ll}
\hline Reference state $w_{\text {ref }}=4 \%$ & Function of correction \\
\hline$k_{\text {ref }}^{(0)}=2.29 \times 10^{4}$ & $b^{(0)}=1-0.055 \times(w-4)$ \\
$k_{\text {ref }}^{(1)}=1.52 \times 10^{5}$ & $b^{(1)}=1-0.012 \times(w-4)$ \\
$k_{\text {ref }}^{(2)}=1.72 \times 10^{6}$ & $b^{(2)}=1-0.012 \times(w-4)$ \\
$k_{\text {ref }}^{(3)}=8.9 \times 10^{4}$ & $b^{(3)}=1-0.059 \times(w-4)$ \\
$\eta_{\text {ref }}^{(1)}=4.57 \times 10^{8}$ & $a^{(1)}=1-0.015 \times(w-4)$ \\
$\eta_{\text {ref }}^{(2)}=2.47 \times 10^{8}$ & $a^{(2)}=1-0.20 \times(w-4)$ \\
$\eta_{\text {ref }}^{(3)}=1.86 \times 10^{8}$ & $a^{(3)}=1-0.17 \times(w-4)$ \\
\hline
\end{tabular}

Table II gives the values of spring modulus $k_{\text {ref }}^{(p)}(\mathrm{MPa})$ and the dashpot viscosities $\eta_{\text {ref }}^{(q)}(\mathrm{MPa} s)$ at the reference state $\left(w_{\text {ref }}=4 \%\right)$ as well as the functions of correction $b^{(p)}$ and $a^{(q)}$.

Figure 8 shows the experimental total strain and that simulated numerically.

We can note that the first drying under stress does not generate a great increase in the viscoelastic strain, unlike the first moistening under load where the moisture content never before reached is now exceeded. With this test, the initial maximum moisture content is never exceeded at the later moistening; also one does not observe an acceleration of the viscoelastic strain. After unloading, residual strains appear, a small part of which will be covered during the first moistening. The residual strains are due to the accumulation of the shrinkage-swelling strains, which is explained by a difference between the shrinkage and the swelling during a moisture cycle. The

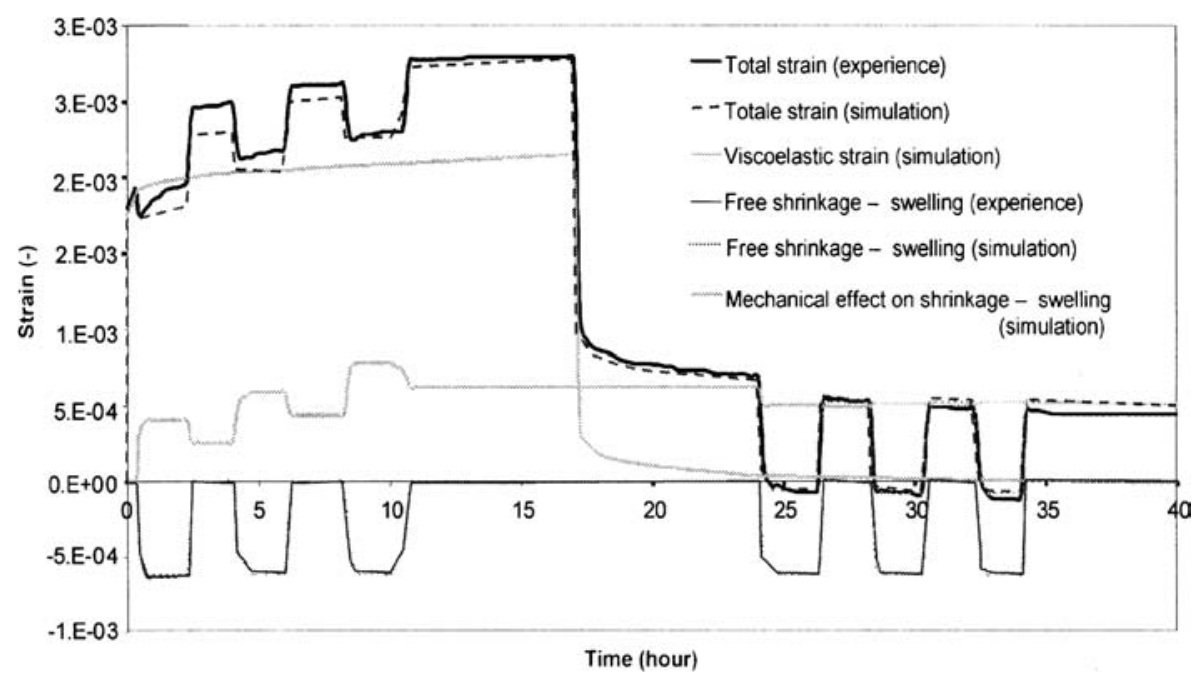

Figure 8. Results of the simulation. 
comparison of the numerical and experimental results shows differences that can be explained by different argumentations. Comparing the apparent creep evolution and the viscoelastic strains, we can suspect a bad interpolation of creep functions. The main difficulty is to predict the viscoelastic behavior for the totality of experimentation by interpolating the viscoelastic properties during a small creep phase. The moisture content cycles are assumed by supposing a bijective relation with the temperature and the relative humidity of the environment. In this case, the hysteresis phenomena is not taken into account in the computation of moisture content. This consideration can explain difficulties in simulating the free strain variations. Finally, in this second experimentation, the relative high stress level (32MPa) can induce a nonlinear viscoelastic behavior and can amplify the nonrecoverable strains.

\section{Conclusions and Perspectives}

While based on realistic thermodynamic principles, this original formulation enables us to better understand the mechanisms involved in the moistening and drying process of wood material. It makes it possible to show that most of the phenomena called mechanosorption are especially of viscoelastic nature, the delayed effects of which grow blurred over time (Hanhijarvi and Hunt, 1998). It is possible to isolate the various phenomena concerned in the process, which it is not possible with a single test. In order to have a realistic simulation, it is necessary to apply an identification strategy to the tested specimen in order to calculate, separately, the mechanical characteristics in a dry and wet state. The great difficulty of this work is to isolate the various effects by avoiding inopportune couplings. Lastly, for application of this tool to elements of larger structural size, it is of primary importance to take into account the principle of diffusion, which generates a gradient, damping and a temporal dephasing between the internal moisture and the external climatic variations. It is thus necessary to develop a coupled model of thermal and hydrous transfer whose diffusion parameters depend on the stress state, internal moisture and the temperature in the material.

\section{Acknowledgements}

The authors gratefully acknowledge Dr P. Navi and Dr V. Pittet at the Laboratory of Building Material, Ecole Polytechnique Fédérale Lausanne (Switzerland), for their collaboration. Thanks are also due to the Limousin Region for the financial support to this study through the European Fund for the Development of Rural Regions (PDZR).

\section{Note}

\footnotetext{
${ }^{1}$ Wood viscoelastic behavior is nonaging when its temperature and its moisture content are constant. It is linear for low stress level
} 


\section{References}

Arfaoui, M., Formulation de lois de comportement viscoélastique: aspects linéaires et non linéaires, PhD Thesis, Université Limoges, France, 2000.

Armstrong, L.D. and Kingston, R.S.T., 'Effect of moisture changes on creep in wood', Nature 185, 1960, 862-863.

Bazant, Z.P., 'Thermodynamics of solidifying or melting viscoelastic material', J. Eng. Mech. Div. 105(EM6), 1979, 933-955.

Bazant, Z.P., 'Rate-type concrete creep law with reduced time', J. Eng. Mech. 110(3), 1984, 329340.

Bazant, Z.P., 'Constitutive equation of wood at variable humidity and temperature', Wood Sci. Technol. 19, 1985, 159-177.

Bazant, Z.P. and Carol, I., 'Viscoelasticity with aging caused by solidifying of nonaging constituent', J. Eng. Mech. Div. 119(11), 1993, 2252-2269.

Bazant, Z.P. and Huet, C., 'Thermodynamic functions for aging viscoelasticity: Integral form without internal variables', Int. J. Solids Struct. 36, 1999, 3993-4016.

Bengtsson, C., Mechanosorptive creep in wood-Experimental studies of the influence of material properties, PhD Thesis, Chalmers University, Goteborg, Sweden, 1999.

Cariou, J.L., Caractérisation d' un matériau viscoélastique anisotrope: le bois, $\mathrm{PhD}$ Thesis, Université Bordeaux I, France, 1987.

Dubois, F., Modélisation du comportement mécanique des milieux viscoélastiques fissurés: Application au matériau bois, PhD Thesis, Université Limoges, France, 1997.

Genevaux, J.M. and Guitard, D., 'Anisotropie du comportement différé: Essai de fluage à température croissante d'un bois de peuplier', Acte du Colloque Scientifique Européen du Groupement Scientifique Rhéologie du Bois, Bordeaux, France, 8-9 June 1988.

Ghazlan, G., Caperaa, S., and Petit, C., 'An incremental formulation for the linear analysis of thin viscoelastic structures using generalized variables', Int. J. Numer. Method Eng. 38, 1988, 33153333.

Gril, J., Une modélisation du comportement hygro-rhéologique du bois à partir de sa microstructure, PhD Thesis, Université Paris 6, France, 1988.

Grossman, P.U.A., 'Requirements of models that exhibit mechanosorptive behavior', Wood Sci. Technol. 10, 1976, 163-168.

Hanhijarvi, A., Modelling of creep strain mechanism in wood, PhD Thesis, Espoo University, Finland, 1995.

Hanhijarvi, A., 'Advances in the knowledge of the influence of moisture changes on the long-term mechanical performance of timber structures', Mater. Struct. 33, 2000, 43-49.

Hanhijarvi, A. and Hunt, D., 'The viscoelasticity of wood at varying moisture content', Wood Sci. Technol. 32, 1998, 57-70.

Huet, C., Quelques aspects du comportement thermo-hygro-viscoélasticité du bois Acte du Colloque Scientifique Européen du Groupement Scientifique Rhéologie du Bois, Bordeaux, France, 8-9 June 1988.

Hunt, D., 'Creep trajectories for beech during moisture changes under load', J. Mater. Sci. 19, 1984, $1456-1467$.

Hunt, D., 'Longitudinal moisture-shrinkage coefficients of softwood at the mechanosorptive creep limit', Wood Sci. Technol. 22, 1988, 199-210.

Hunt, D., 'A unified approach to creep of wood', Proc. R. Soc. Lond. Acad. 455, 1999, 4077-4095.

Joyet, P., Comportement différé du matériau bois dans le plan transverse sous des conditions hydriques évolutives, PhD Thesis, Université Bordeaux I, France, 1992.

Juerkiewiez, B., Analyse et modélisation du comportement différé des ouvrages constitués de plusieurs matériaux, PhD Thesis, Université Blaise Pascal - Clermont II, France, 1996. 
Kelley, S.S., Rials, T.G., and Glasser, W.G., 'Relaxation behaviour of the amorphous components of wood', J. Mater. Sci. 22, 1987, 617-624.

Lassere, B., Modélisation themo-hydro-mécanique du comportement différé de poutres de structure en bois., PhD Thesis, Université Bordeaux I, France, 2000.

Le Govic, C., Rouger, F., and Felix, B., 'Approche d'un modèle multitransitions paraboliques pour le fluage du bois', Acte du Colloque Scientifique Européen du Groupement Scientifique Rhéologie du Bois, Bordeaux, France, 8-9, June 1988.

Leicester, R.H., 'A rheological model for mechanosorptive deflections of beams', Wood Sci. Technol. 5, 1971, 211-220.

Lenth, C.A., Wood material behaviour in severe environments, PhD Thesis, Virginia Polytechnic Institute and State University, Blacksburg, 1999.

Martensson, A., Mechanical behaviour of wood exposed to humidity variation, $\mathrm{PhD}$ Thesis, Lund University, Sweden, 1992. Report TVBK-1006.

Morlier, P., Creep in timber structures. RILEM Report 8, 1994.

Mukuday, S. and Yata, S., 'Modeling and simulation of viscoelastic behavior (tensile strain) of wood under moisture change', Wood Sci. Technol. 20, 1986, 335-348.

Pittet, V., Etude expérimentale des couplages mécano-sorptifs dans le bois soumis à des variations hygrométriques contrôlées sous chargements de lonque durée, $\mathrm{PhD}$ Thesis, Ecole Polytechnique Fédérale Lausanne, Suisse, 1996.

Randriambololona, H. and Dubois, F., Modélisation numérique du comprotement viscoélastique linéaire assouplissant et rigidifiant: Application au bois. Research Report 10, L3McGC, Egletons, France, 2001.

Randriambololona, H., Dubois, F., and Petit, C., 'Modeling of viscoelastic behaviour of wood material subjected to environmental variation', Proceedings of the International Conference of the European Society for Wood Mechanics, ICESWM'01, EPFL - LMC, Lausanne, Suisse, 19-20 April 2001.

Randriambololona, H., Dubois, F., and Petit, C., 'Modélisation du comportement viscoélastique du matériau bois dans un environnement variable', Actes du Colloque Ouvrage et systéme en Service, COSS'01, EUDIL, Lille, France, 3-4 May 2001.

Randriambololona, H., Dubois, F., and Petit, C., 'Modélisation du comportement mécanique différé du bois en environment variable', Rev. Francaise Génie Civil 6(7-8), 2002, 1347-1371.

Ranta-Maunus, A., 'The viscoelasticity of wood at varying moisture content', Wood Sci. Technol. 9, 1975, 189-205.

Salin, J.G., 'Numerical prediction of checking during timber drying and a new mechanosorptive creep model', Holz als Roh und Werkstoff 50, 1992, 195-200.

Salmen, N.L. and Back, E.L., 'The influence of water on the glass transition temperature of cellulose, TAPPI J. 60, 1977, 137-140.

Schaffer, E.L., 'Modeling the creep of wood in a changing moisture environment', Wood Fiber 3, 1972, 232-235.

Tissaoui, J., Effect of long-term creep on the integrity of modern wood structures, PhD Thesis, Virginia Polytechnic Institute and State University, Blacksburg, 1996.

Toratti, T., Creep of timber beams in variable environment, $\mathrm{PhD}$ Thesis, Helsinki University of Technology, Finland, 1992.

van Der Put, T.C.A.M., Deformation and damage processes in wood, PhD Thesis, Delft University, The Netherlands, 1989.

Zienkiewicz, O.C., La méthode des éléments finis, McGraw-Hill, New York, 1979.

Zienkiewicz, O.C., Watson, M., and King, I.P., 'A numerical method of viscoelastic stress analysis', Int. J. Mech. Sci. 10, 1968, 807-827. 